\title{
ON ELECTRONS IN QUANTUM CHAOS STATE IN DOPED FULLERENE CRYSTAL
}

\author{
A. Koper and M. MUCha \\ Institute of Physics, Adam Mickiewicz University \\ Umultowska 85, 61-614 Poznań, Poland
}

We show that band electrons in $\mathrm{A}_{n} \mathrm{C}_{60}$ crystal $\left(\mathrm{C}_{60}\right.$ fullerene doped with alkali ions A) are in highly chaotic quantum state. We describe intensity of the chaos by means of the Shannon information entropy, which we calculate using single particle Bloch functions. The entropy provides a quantitative measure of scars as well as degree of electrons delocalization in gaps between $\mathrm{C}_{60}$ molecules. Implications of our results for conductivity in $\mathrm{A}_{3} \mathrm{C}_{60}$ are discussed.

PACS numbers: $05.45 .+b, 61.48 .+c$

\section{Introduction}

In this paper we show that electrons from neighborhood of the Fermi surface in molecular crystals $\mathrm{A}_{n} \mathrm{C}_{60}$, where A denotes alkali metal (K, Rb), are in highly chaotic quantum state, whose origin is of both geometrical and band nature.

Phenomenological explanation of geometrical chaos is as follows. Let us assume that peak of fully occupied band $h_{\mathrm{u}}$ of non-doped crystal $\mathrm{C}_{60}$ is zero on energy scale. If we add ions $A$, together with additional electrons (thermodynamically stable values of $n$ are of order $3,4,6$ ), to such a crystal, then according to the band theory $[1,2]$, a new Fermi level for the electrons $E_{\mathrm{F}} \approx 1.5 \mathrm{eV}$ will be lying in the bands group labeled as $t_{1 \mathrm{u}}$. The bands $h_{\mathrm{u}}$ and $t_{1 \mathrm{u}}$ are separated by energy gap of $\approx 1 \mathrm{eV}$, and the band width of $t_{1 \mathrm{u}}$ is of order $\approx 0.5 \mathrm{eV}$. When we express the energy in terms of the de Broglie electrons wavelengths (we assume purely kinematic growth of energy in molecular crystal) we get $\lambda_{F} \approx 10 \AA$. This means that the electrons in the neighborhood of the Fermi surface in doped molecular crystal can "feel" geometry of whole $\mathrm{C}_{60}$ molecules, since their diameter $D$ is of the same order $D \approx 10 \AA$. In such a case these electrons, in quasi-free electron approximation, can be treated as billiard balls in three-dimensional quantum billiard, where the scattering centers role is played by heavy $\mathrm{C}_{60}$ molecules.

The geometrical quantum chaos described above gives contribution to chaos of the $t_{1 \mathrm{u}}$-electrons. Obviously not all energy levels covered by this scattering model are admissible in $\mathrm{A}_{n} \mathrm{C}_{60}$ crystals since their band structure $h_{\mathrm{u}}, t_{1 \mathrm{u}}, t_{1 \mathrm{~g}} \ldots$ is clearly preserved. 
The band chaos is of purely quantum origin. It appears in all those crystals, where band electrons are partly localized within the elementary cell. In the case of $\mathrm{A}_{n} \mathrm{C}_{60}$ systems it should be particularly sharply visible because of molecular structure. Computer calculations [2] show that potential of a $\mathrm{C}_{60}$ molecule in the crystal can be modeled by attracting spherically symmetric well. Top of the well is on the spherical surface of diameter $D \approx 10 \AA$ and its depth is of $\approx 3 \AA$. Therefore, the electrons from $t_{1 \mathrm{u}}$ band will be partly localized in energy wells of $\mathrm{C}_{60}$ centers, which are also the nodes of crystal lattice. This together with the translational symmetry condition (the lattice has the fcc structure) should lead to strong oscillation of wave functions in nodes neighborhood and in consequence to quantum chaos.

We describe both kinds of quantum chaos by means of the Shannon entropy $S_{\nu}(k)$ calculated from single particle Bloch functions. It can be shown that localization length $l_{\nu}(k)$ of the Bloch function $\psi_{\nu, k}(x)$ is proportional to $\exp S_{\nu}(k)$ in $\mathrm{C}_{60}$ nodes neighborhood [3]. The Shannon entropy can be treated as an indicator of "fitness" of Berry's conjecture [4], i.e. large value of the entropy indicates correctness of the conjecture.

\section{Chaos entropy}

According to the Bloch theorem the electron's quantum state $\psi_{\nu, \boldsymbol{k}}$ and its energy $E_{\nu}(k)$ (in $\nu$-th band for vector $k$ from the Brillouin zone), in translationally invariant crystal, can be determined from the Schrödinger equation

$$
\left[-\frac{\hbar^{2}}{2 m} \nabla^{2}+\sum_{i} V\left(x-R_{i}\right)\right] \psi_{\nu, k}(x)=E_{\nu}(k) \psi_{\nu, k}(x),
$$

where $V\left(\boldsymbol{x}-\boldsymbol{R}_{\boldsymbol{i}}\right)$ denotes potential around $i$-th lattice node whose location is indicated by vector $\boldsymbol{R}_{i}$ and $m$ is the electron mass. The sum runs over all lattice nodes. In the case of finite crystals of volume $\Omega$ we assume periodic boundary conditions.

As it is commonly known the functions $\psi_{\nu, k}(x)$ can be decomposed into plane waves

$$
\psi_{\nu, \boldsymbol{k}}(\boldsymbol{x})=\Omega^{-1 / 2} \sum_{n} \mathrm{e}^{\mathrm{i}\left(\boldsymbol{k}+\boldsymbol{K}_{n}\right) \boldsymbol{x}} \phi_{\nu}\left(\boldsymbol{k}+\boldsymbol{K}_{n}\right) .
$$

Summation in the above formula runs over all vectors $\boldsymbol{K}_{n}$ of the reciprocal lattice. From analysis of normalization condition $\sum_{n}\left|\phi_{\nu}\left(k+K_{n}\right)\right|^{2}=1$ we get the following interpretation of the Fourier coefficients: $\phi_{\nu}\left(k+K_{n}\right)$ is an amplitude of probability that the electron is moving within the crystal in the direction of $k+K_{n}$ vector. An electron described by the function $\psi_{\nu, k}$ can be considered as being in chaos state when the amplitudes $\left|\phi_{\nu}\left(k+\boldsymbol{K}_{n}\right)\right|$ are of the same order for all directions $\boldsymbol{K}_{n}$. This statement is an analogue of Berry's conjecture, which says that quantum eigenstates of classical ergodic systems locally look like random superposition of plane waves [4]. If this is the case then the wave function $\psi_{\nu, k}(x)$ should have scars in real space [5]. The more scars the more delocalized is the function. Since description of wave function scars (in particular in the three-dimensional space $\boldsymbol{x}$ ) 
by means of graphs is rather inconvenient we will use the notion of the Shannon entropy in a state $\psi_{\nu, \boldsymbol{k}}$ which is defined as follows:

$$
S_{\nu}(k)=-\sum_{n}\left|\phi_{\nu}\left(k+\boldsymbol{K}_{n}\right)\right|^{2} \log \left|\phi_{\nu}\left(k+\boldsymbol{K}_{n}\right)\right|^{2} .
$$

The entropy $S_{\nu}(k)$ is non-negative and in the case of maximal chaos it behaves like $\log N$, where $N$ is number of bands. Using the entropy $S_{\nu}(k)$ and energy $E_{\nu}(k)$ we can define common density partition function for energy and entropy in the Brillouin zone (BZ) as

$$
\rho(E, S)=\sum_{\nu} v_{\mathrm{BZ}}^{-1} \int_{\mathrm{BZ}} \mathrm{d} k \delta\left(E-E_{\nu}(k)\right) \delta\left(S-S_{\nu}(k)\right),
$$

where $v_{\mathrm{BZ}}$ denotes the volume of $\mathrm{BZ}$ and $\delta$ is the Dirac function. The density $\rho(E, S)$ takes high values where there is high density of states of energy $E$ and simultaneously high density of states of entropy $S$. High value of entropy means high delocalization of electron states in gaps between lattice nodes.

\section{Results and conclusions}

We solve stationary Schrödinger equation for three model potentials:

(i) For description of the geometric chaos we use spherical potential $V_{G}(x)=$ $V_{\mathrm{G}} \theta\left(R^{2}-x^{2}\right)$, where $\theta$ is the Heaviside step function and $R=D / 2=3.55 \AA$ is the radius of $\mathrm{C}_{60}$ molecule. The potential $V_{\mathrm{G}}=3 \mathrm{eV}$ was derived from computer simulation as a condition of effective scattering of particles with wavelength $D$.

(ii) For description of the band chaos we use the function $V_{B}(x)=$ $V_{B}\left\{\theta\left[(R+\Delta R)^{2}-x^{2}\right]-\theta\left(R^{2}-x^{2}\right)\right\}$, where $\Delta R=3 \AA$ and potential $V_{B}=$ $-4 \mathrm{eV}$.

(iii) For description of the mixed case of bands and geometrical chaos we use the function $V(x)=V_{B}(x)+V_{\mathrm{G}}(x)$.

In order to simplify the calculations we assume that the lattice is sc and the lattice constant is $a=10 \AA$ i.e. the real distance between the nearest neighbors $\mathrm{C}_{60}-\mathrm{C}_{60}$. The integrals $\rho(E, S)$ were calculated using $46 \mathrm{k}$ of special (high symmetry) points in BZ. The graphs of $\rho(E, S)$ are normalized. The beginning of energy scale was set at the peak of fully occupied band $h_{\mathrm{u}}$. Calculations were carried out for clusters of the size $5 \times 5 \times 5$ in the reciprocal space which means that we took into account $N=125$ bands.

Geometrical quantum chaos dominates for large non-negative energies (cf: Fig. 1a), whereas in case of negative energies the band chaos dominates (cf. Fig. 1b). The geometrical chaos favors increase in general quantum entropy. Band of higher chaos are moved in the direction of increased entropy (cf. Fig. 1b and c).

Besides Berry's conjecture there exists spectral conjecture (cf. [6]) but its direct application to our case is impossible since our system has the continuous spectrum. We expect that generalization of spectral approach to continuous case should lead to appearance of (density) partition functions of group velocity which are used in formulas describing electron transport in crystals.

High quantum chaos for $\mathrm{A}_{3} \mathrm{C}_{60}$ molecular crystal (where expected Fermi energy levels are marked in Fig. 1) can be responsible for bad conductivity and 

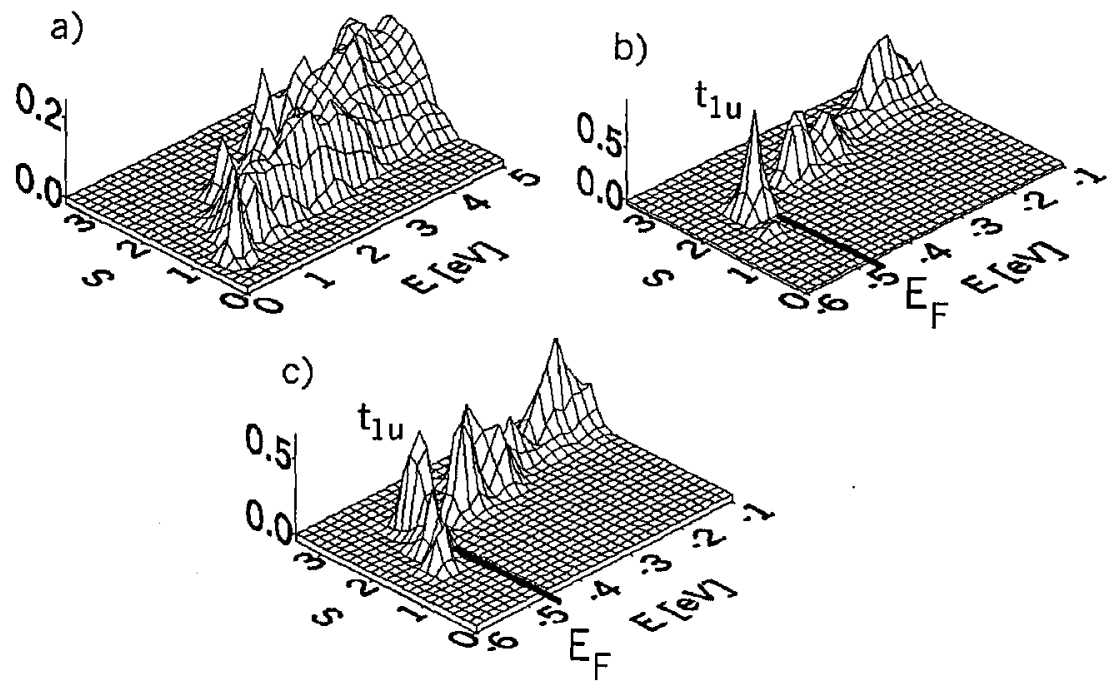

Fig. 1. Graphs of density $\rho(E, S) / N$, where $N$ is the number of bands. $E_{\mathrm{F}}$ denotes expected Fermi energy level for $\mathrm{A}_{3} \mathrm{C}_{60}$ crystal. (a) Geometrical chaos. (b) Bands chaos. (c) Bands and geometrical chaos. The peaks (left to right) belong to bands $t_{1 \mathrm{u}}, t_{1 \mathrm{~g}}$, etc.

linear dependence of resistivity on temperature of the system. This result (high quantum chaos) agrees well with expectation that the electron transport in $\mathrm{A}_{3} \mathrm{C}_{60}$ can be controlled by quantum critical point [7]. Other implications of the model are discussed elsewhere [8].

\section{References}

[1] S.C. Erwin, in: Buckminsterfullerenes, Eds. W.E. Billups, M.A. Ciufolini, VCH Publishers, New York 1993, p. 217.

[2] N. Troullier, J.L. Martins, Phys. Rev. B 46, 1754 (1992).

[3] F.M. Izrailev, Phys. Lett. A 134, 13 (1988).

[4] M.V. Berry, J. Phys. A 10, 2083 (1977).

[5] E.J. Heller, Phys. Rev. Lett. 53, 1515 (1984).

[6] O. Bohigas, M.J. Giannoni, C. Schmit, Phys. Rev. Lett. 52, 1 (1984).

[7] V.J. Emery, S.A. Kivelson, V.N. Muthukumar, preprint avoilable on http://xxx.lanl.gov, cond-mat/9901270.

[8] A. Koper, M. Mucha, Acta Phys. Pol. A 97, 233 (2000). 